\title{
Dissociable contributions of thalamic nuclei to recognition memory: novel evidence from a case of medial dorsal thalamic damage
}

\author{
Rachel N. Newsome, ${ }^{1,2,3,6}$ Alexandra N. Trelle, ${ }^{4,6}$ Celia Fidalgo, ${ }^{1}$ Bryan Hong, ${ }^{1}$ \\ Victoria M. Smith, ${ }^{1,3}$ Alexander Jacob, ${ }^{1}$ Jennifer D. Ryan, ${ }^{1,2}$ R. Shayna Rosenbaum, ${ }^{2,3}$ \\ Rosemary A. Cowell, ${ }^{5}$ and Morgan D. Barense ${ }^{1,2}$ \\ ${ }^{1}$ Department of Psychology, University of Toronto, Toronto, Ontario, M5S 3G3, Canada; ${ }^{2}$ Rotman Research Institute, Baycrest, Toronto, \\ Ontario, M6A 2E1, Canada; ${ }^{3}$ Departments of Psychology and Biology, Centre for Vision Research, York University, Toronto, Ontario, \\ M3J 1P3, Canada; ${ }^{4}$ Department of Psychology, University of Cambridge, Cambridge CB2 1 TN, United Kingdom; ${ }^{5}$ Department of \\ Psychology, University of Massachusetts, Amherst, Massachusetts 01003, USA
}

\begin{abstract}
The thalamic nuclei are thought to play a critical role in recognition memory. Specifically, the anterior thalamic nuclei and medial dorsal nuclei may serve as critical output structures in distinct hippocampal and perirhinal cortex systems, respectively. Existing evidence indicates that damage to the anterior thalamic nuclei leads to impairments in hippocampal-dependent tasks. However, evidence for the opposite pattern following medial dorsal nuclei damage has not yet been identified. In the present study, we investigated recognition memory in NC, a patient with relatively selective medial dorsal nuclei damage, using two object recognition tests with similar foils: a yes/no (YN) test that requires the hippocampus, and a forced choice corresponding test (FCC) that is supported by perirhinal cortex. NC performed normally in the YN test, but was impaired in the FCC test. Critically, FCC performance was impaired only when the study-test delay period was filled with interference. We interpret these results in the context of the representational-hierarchical model, which predicts that memory deficits following damage to the perirhinal system arise due to increased vulnerability to interference. These data provide the first evidence for selective deficits in a task that relies on perirhinal output following damage to the medial dorsal nuclei, providing critical evidence for dissociable thalamic contributions to recognition memory.
\end{abstract}

The medial temporal lobe (MTL) has long been implicated in recognition memory, and existing evidence from humans and animals with focal MTL damage suggests a division of labor between MTL subregions. The distinct contribution of each of these regions to recognition memory has been conceptualized in a number of different ways (Aggleton and Brown 1999; Squire et al. 2007; Cowell et al. 2010). For example, this distinction has often been couched in terms of the different mnemonic processes supported by each region, such that hippocampus supports recollection of contextual details associated with an item's prior occurrence, whereas perirhinal cortex (PRC) supports the acontextual feeling of familiarity that an item has been encountered previously (Aggleton and Brown 1999). More recently, however, there is increasing agreement that the role of these regions may be better understood in terms of the nature of the information that they represent and the computations that they perform (Davachi 2006; Bussey and Saksida 2007; Diana et al. 2007; Cowell et al. 2010; Graham et al. 2010; Norman 2010; Ranganath 2010; Sadeh et al. 2014; Ross et al. 2017). For example, the representational-hierarchical account proposes that the PRC supports representations at the level of individual items (i.e., the conjunction of features composing an object), whereas the hippocampus supports more complex representations of relations between items and their spatio-temporal

${ }^{6}$ These authors contributed equally to this work.

Corresponding author: rachel.newsome@utoronto.ca

Article is online at http://www.learnmem.org/cgi/doi/10.1101/lm.045484. 117. context (Cowell et al. 2010, see also Ranganath 2010, Sadeh et al. 2014; for similar views, see Lee et al. 2012).

Despite the considerable emphasis on MTL contributions to recognition memory over the years, mounting evidence suggests that structures outside of the MTL are also critical for supporting recognition memory. In particular, medial diencephalic pathology leads to impairments in recognition memory similar to those caused by focal MTL lesions (Aggleton et al. 2010, 2011; Mitchell and Chakraborty 2013). The regions that are thought to play a critical role in memory include the mammillary bodies and the anterior thalamic nuclei (ATN), which receive projections from the hippocampus via the fornix (Aggleton et al. 1986), as well as the medial dorsal thalamic nuclei (MDN), which receive projections from PRC (Fig. 1; Amaral 1999; Saunders et al. 2005; Aggleton et al. 2011). These distinct neuroanatomical connections have led to the proposal that thalamic nuclei make dissociable contributions to recognition memory, as described by the multieffect multinuclei model (MEMN) proposed by Aggleton et al. (2011). In particular, this model suggests that the mammillary bodies and the ATN form part of an extended hippocampal system that is necessary for recall and recollection-based recognition, whereas

2018 Newsome et al. This article is distributed exclusively by Cold Spring Harbor Laboratory Press for the first 12 months after the full-issue publication date (see http://learnmem.cshlp.org/site/misc/terms.xhtml). After 12 months, it is available under a Creative Commons License (AttributionNonCommercial 4.0 International), as described at http://creativecommons. org/licenses/by-nc/4.0/. 

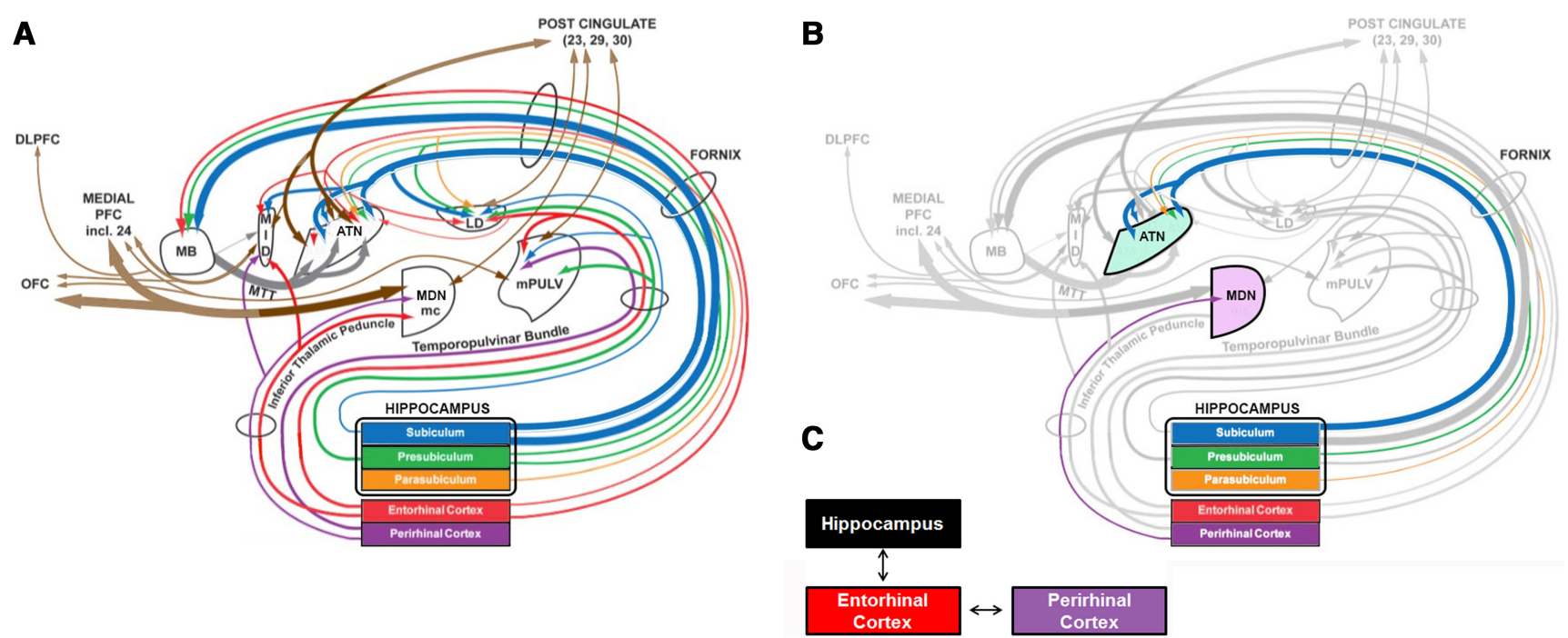

Figure 1. (A) Depiction of the thalamic connections to the medial temporal lobe, modified from Aggleton et al. (2011). (B) Modified figure depicting the key thalamic connections in the present study. Specifically, the anterior thalamic nuclei (ATN) have connections to the hippocampus, and the medial dorsal nucleus (MDN) has connections to the perirhinal cortex. Case NC described here has damage to the MDN. (C) Simplified depiction of connections within the MTL (modified from Murray and Wise 2012). Note that because NC's perirhinal cortex is intact, we hypothesize that object-level representation can still reach the hippocampus, enabling his intact YN recognition memory.

the MDN and PRC form a distinct system that supports familiaritybased recognition (Aggleton and Brown 1999; Aggleton et al. 2011).

Early evidence for this proposal comes from studies of patients with selective damage to the fornix, mammillary bodies or ATN who exhibited severe deficits in recall performance, coupled with relatively spared recognition (Carlesimo et al. 2007; Tsivilis et al. 2008; Vann et al. 2008, 2009). For example, Carlesimo et al. (2007) found that a patient with a bilateral ATN lesion, with damage extending bilaterally to the mammillothalamic tract, exhibited impaired recollection with intact familiarity using remember/ know judgments and receiver operating characteristic (ROC) curves. Critically, this behavioral dissociation is analogous to that observed following selective hippocampal lesions (Holdstock et al. 2002; Yonelinas 2002). Similarly, extensive damage to the ATN and partial damage to the MDN produced severe deficits in recollection but only minimal deficits in familiarity, as assessed through both remember/know judgments and ROC curves (Kishiyama et al. 2005).

Although these findings are consistent with dissociable contributions of distinct thalamic nuclei to recognition memory, an important part of the story is missing: to our knowledge, there are no published studies involving humans showing that damage to the MDN produces similar deficits as that observed following focal PRC damage. The scarcity of such selective lesions has made testing the full double dissociation difficult. However, evidence consistent with this proposal has been obtained through studies of nonhuman primates. For example, lesions of the MDN that spare the ATN produced deficits in object recognition memory that are analogous to those observed following rhinal cortex lesions, albeit to a lesser degree (Zola-Morgan and Squire 1985; Parker et al. 1997). Moreover, disconnection of the MDN and rhinal cortex impaired object recognition memory to a similar degree as did disconnection of the prefrontal cortex and rhinal cortex, an effect that was not observed following amygdala ablation or fornix transection (Parker and Gaffan 1998). These findings in nonhuman primates raise the possibility that selective damage to the MDN in humans may produce similar deficits to PRC lesions, just as damage to the ATN produces deficits that resemble those observed following hippocampal lesions.
The current study explores this possibility by assessing recognition memory performance in NC, a person who experienced a thalamic stroke resulting in damage to the MDN bilaterally, leaving the ATN volumetrically unaffected (D'Angelo et al. 2015). Specifically, we adopted a method introduced by Holdstock et al. (2002) that is well suited for characterizing memory deficits in amnesic patients, in that it avoids many of the confounds associated with the use of subjective measures of the phenomenological experiences associated with remembering. This method compares performance in an object recognition memory test with perceptually similar foils across two test formats: a standard yes/no (YN) test format in which targets and foils are presented individually, and a forced choice corresponding (FCC) test format, wherein targets and corresponding foils are presented simultaneously.

\section{The role of test format in recognition memory deficits}

A growing body of evidence suggests that when targets and foils share a high degree of overlap, the neural mechanisms necessary to support object recognition memory vary according to the test format with which memory is assessed. In particular, existing proposals state that performance in a $\mathrm{YN}$ test requires the hippocampus, whereas performance in a FCC test can be supported by PRC (Holdstock et al. 2002; Norman 2010). The contributions of the hippocampus and PRC to performance can be summarized in terms of either the "processes" (recollection versus familiarity) or the "representations" (item-level versus item-in-context level) necessary to support performance in each test format. Both explanations make similar predictions and agree that the dissociation between test formats arises because targets and foils with a high degree of feature overlap produce highly overlapping distributions of familiarity/memory strength for targets and foils, but they differ in terms of the underlying mechanism thought to be driving the effects.

According to a process-based explanation, the FCC test is supported by PRC because it supports judging the relative familiarity between exemplars (Migo et al. 2009, 2014; Norman 2010). For any stimulus set, there are variable levels of absolute familiarity (or "memory strength") across stimuli, driven by preexperimental 
or inherent stimulus factors other than study exposure. This variability does not pose a problem in the FCC format, because the similarity of the two items ensures that their preexperimental familiarity is similar: any familiarity difference between them is therefore a reliable index of which object appeared in the study phase (Migo et al. 2009, 2014; Norman 2010). In contrast, the YN test is said to require a different process-recollection-to reinstate a precise, pattern-separated representation of the object that is sufficiently distinct from similar items to be endorsed only when it matches the studied object; this process depends on hippocampus (Holdstock et al. 2002; Norman 2010). Consistent with this proposal, healthy participants who were instructed to rely on stimulus familiarity to support performance were impaired in YN, but performed normally in FCC (Migo et al. 2009).

Alternatively, we can consider differences in the complexity of the representations necessary to perform discriminations of targets and foils with overlapping features across test formats. According to a representational account, the FCC test is supported by PRC because it contains object-level representations in the absence of contextual information, whereas the hippocampus contains the representation of an item bound to its original spatiotemporal context (Cowell et al. 2010; McTighe et al. 2010; Martin et al. 2013; Yeung et al. 2013). Here, the key difference between test formats is the necessity for retrieving a representation of the object in its original studied context, a distinction that has also been proposed by others (e.g., Migo et al. 2009). When assessing memory using object-level representations devoid of temporal context, the variability in preexperimental familiarity of stimuli does not pose a problem in the FCC format, because the small but reliable familiarity difference between the representations of those two objects indicates which item was studied. Thus, performance in the FCC test is supported by PRC. In contrast, the YN format presents only one object, so that variable levels of preexperimental stimulus familiarity make it difficult to use only upon object-level representations to set a criterion across trials that includes all studied items and excludes all unstudied items. Instead, good YN performance requires knowing whether the conjunction of this-item-in-this-experimental-context seems familiar-a representation thought to reside in the hippocampus.

Critically, however, according to a representational account, because features are shared across targets and foils, the nature of the target object representation is relevant for performance. In particular, representations of individual perceptual or semantic features that compose the object will be insufficient to support successful discriminations, as these are likely to be common to both exemplars (e.g., both have a floral pattern, both are spherical, both are beach balls, etc.). Instead, accurate discriminations necessitate a representation of the target object that codes for the complex conjunction of features composing the object, including a combination of perceptual and semantic features. According to the representational-hierarchical framework, these conjunctive object-level representations are supported by PRC, and are critical for overcoming interference inherent in repeating and overlapping features between exemplars and across objects (Cowell et al. 2010; McTighe et al. 2010; Barense et al. 2012; Erez et al. 2016). As a result, PRC damage or dysfunction should impair FCC discrimination between perceptually similar exemplars. Consistent with this proposal, previous work has identified a relationship between PRC gray matter volume and FCC memory performance in individuals with mild cognitive impairment and Alzheimer's disease (Westerberg et al. 2013), suggesting that PRC integrity, and thus the availability of the representations it supports, may be systematically related to FCC performance.

If the ATN and MDN form critical components of distinct hippocampal- and PRC-systems, respectively, selective deficits in performance across test formats may also be observed following lesions to thalamic nuclei. Thus, the first aim of the present experiment was to test the prediction that damage to the MDN results in selective deficits in recognition memory performance that relies critically on PRC output. To this end, we assessed NC's performance in both the YN and FCC test formats of an object recognition test with similar foils. We predicted that NC would be impaired in the FCC test, which relies on PRC output, but perform normally in the YN test, which relies on output from the hippocampus, consistent with the proposal that the MDN is a critical output structure for PRC, as proposed by the MEMN model. Furthermore, in accordance with the representational-hierarchical view, we also predicted that this deficit would not be all or none, but rather dependent on the presence of visual interference.

\section{The role of interference in recognition memory deficits}

The representational-hierarchical view proposes that recognition memory deficits following PRC damage arise due to increased vulnerability to interference (Cowell et al. 2006; Barense et al. 2012). More specifically, a stream of visual input (such as that encountered over a delay) can create interference at the level of individual features, simply because different objects tend to share lower-level features (e.g., shapes, colors, etc.); under these conditions, the intact conjunctive, object-level representations are necessary to support performance. If such representations within the PRC are damaged, performance must be supported by simpler, feature-level representations in more posterior regions of the ventral visual stream. These feature-level representations cannot disambiguate between repeating features shown across different objects; however, the object-level representations usually maintained in PRC are unique to each individual object and can resolve interference from visually similar features. Consistent with this view, previous work has demonstrated that reducing feature-level interference can ameliorate deficits in target-foil discrimination in individuals with compromised PRC integrity, including patients with focal PRC lesions (Barense et al. 2012), amnestic mild cognitive impairment (Newsome et al. 2012), older adults (Yeung et al. 2013; Trelle et al. 2017), aged nonhuman primates (Burke and Barnes 2010; Burke et al. 2011), as well as rodents with focal lesions of the rhinal cortex (Bartko et al. 2010; McTighe et al. 2010).

A relevant source of interference in typical laboratory based tests of recognition memory arises during the study-test delay, which is often filled with cognitive tests that contain distracting visual information. Critically, previous work has demonstrated that minimizing delay-period interference can rescue memory performance following lesions of the rhinal cortex in rodents (McTighe et al. 2010), as well as in patients with MTL damage affecting PRC (Cowan et al. 2005). For example, lesions to rhinal cortex impaired object recognition memory when the study-test delay was filled with interfering information, but had no effect when delay period interference was minimized by putting rodents in a darkened cage (McTighe et al. 2010; see also Romberg et al. 2012). In a similar fashion, robust memory enhancements have been observed in amnesic patients and those with mild cognitive impairment by replacing filled delay periods, during which patients are typically asked to complete other cognitive tasks, with minimal interference conditions, during which participants sit quietly in a darkened room (Cowan et al. 2004, 2005; Dewar et al. 2009, 2012). These observations may be explained by a reduced ability to resolve interference following damage to the visual representational system. That is, the reduced availability of object-level representations may have caused impaired discrimination ability when feature-level visual interference during the delay period was high, but not when it was minimized.

Although the PRC is not volumetrically different in patient $\mathrm{NC}$, structural connectivity data suggest that the MDN may act 
as a key output structure for the PRC (Amaral 1999; Saunders et al. 2005; Aggleton et al. 2011). Accordingly, damage to the MDN, by virtue of blocking information output from PRC, may increase vulnerability to interference in a similar fashion to focal PRC damage. Indeed, previous work in nonhuman primates suggests that lesions of the MDN result in a similar, albeit less severe, pattern of recognition memory deficits to those observed following rhinal cortex lesions. In particular, both MDN and PRC lesions in nonhuman primates impaired recognition memory when long stimulus lists were used (and thus feature-level interference was high), but not when shorter lists of only two stimuli were used (and thus featurelevel interference was minimized) (Parker and Gaffan 1998). Taken together, this evidence raises the possibility that damage to any part of the MDN-PRC system may result in increased vulnerability to interference. However, to our knowledge, this possibility has not been tested previously in a person with selective damage to the MDN. Moreover, the extent to which such effects might be specific to feature-level visual interference, or observed more generally following the presence of any interfering material, remains to be explored.

Thus, the second aim of the present investigation is to test the prediction made by the representational-hierarchical account that recognition memory deficits following damage to the MDN are interference-dependent, and to assess whether the nature of delay period interference modulates the effects of interference on performance. To this end, we assessed NC's recognition memory performance in both the FCC and YN test formats following four types of delay period interference: minimal interference (eyes closed in a dark quiet room), cognitive interference (eyes closed while answering mentally taxing questions), feature-level visual interference (passive viewing of scrambled objects containing features of foil objects), and low-level visual interference (passive viewing of scrambled gray squares) (Fig. 2). We predicted that patient NC would exhibit selective deficits in memory performance in the

\section{Study: Is this object bigger than a shoebox?}
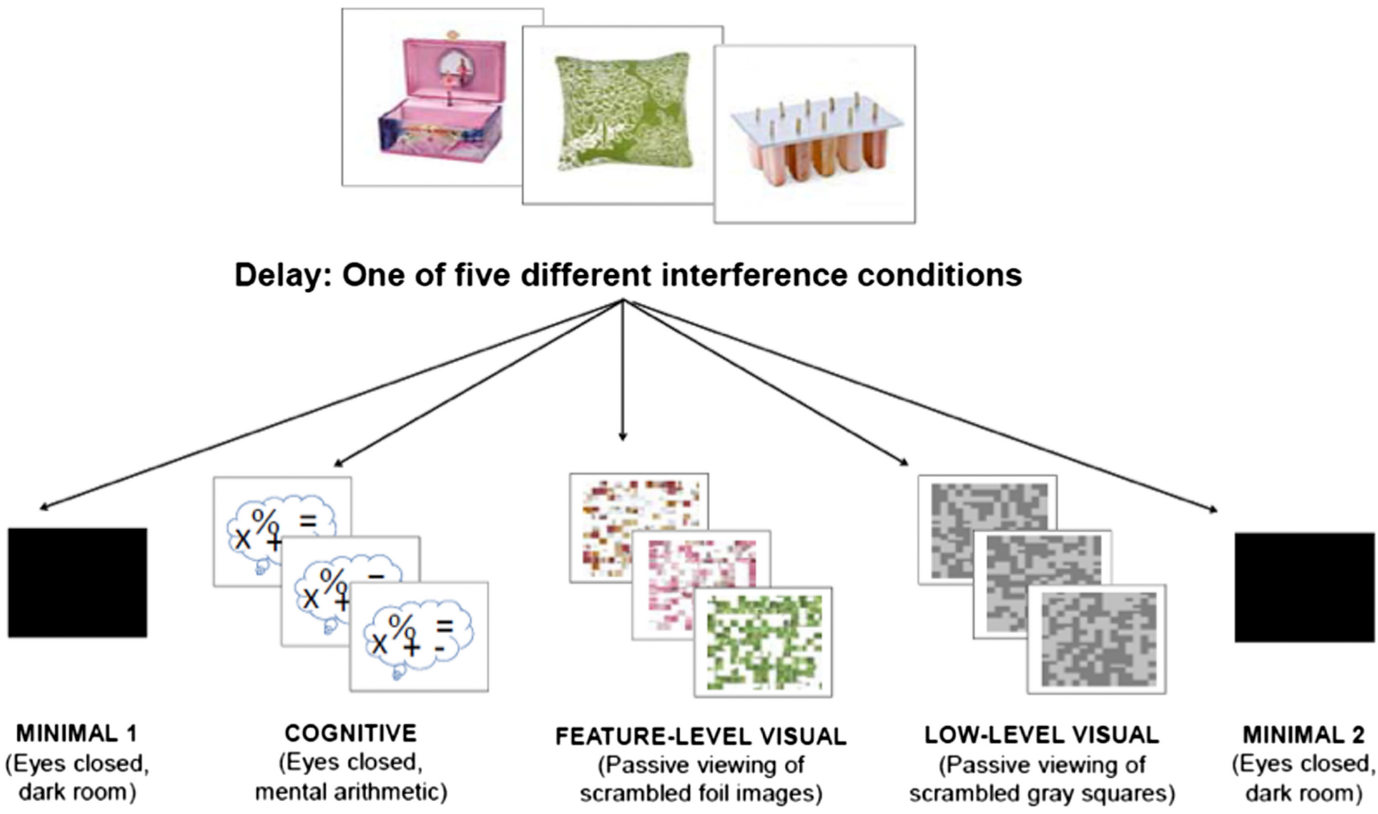

Test: One of two test formats

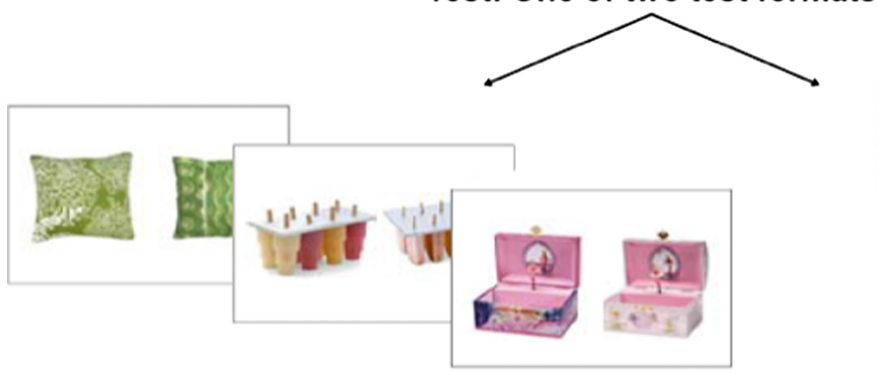

Forced choice:

Which of these did you see previously?

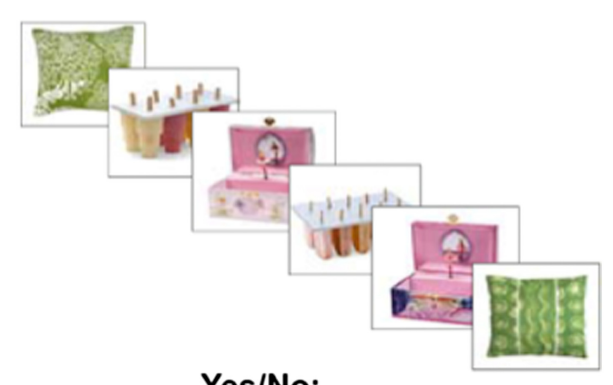

Yes/No:

Did you see this previously?

Figure 2. Experimental protocol. After the study phase, participants were administered one of five interference delay conditions: minimal 1, cognitive, feature-level visual, low-level visual, minimal 2. Following delay, participants completed one of two recognition memory tests: either the forced choice with corresponding foils (FCC) or yes/no (YN). Note that unique stimuli were used in the FCC and YN tasks but are displayed commonly for clarity in the figure. FCC and YN tests were completed in separate testing sessions and in a given session, participants completed all five delay conditions (minimal 1 and 2 were always first and last; the order of the remaining conditions was counterbalanced). 
FCC test that is supported by PRC output, but that the emergence of this impairment would be modulated by the presence and type of interference. More specifically, if the presence of feature-level interference impacts performance, as suggested by existing evidence from humans and animals with focal PRC lesions, NC should be impaired in the FCC test following feature-level visual interference, but perform normally following the low-level visual interference, cognitive interference, and minimal interference delays. In contrast, we predicted that NC's performance in the YN test, which is supported by the ATN-hippocampal system, would not differ from controls across condition, irrespective of the presence or type of delay-period interference.

\section{Results}

\section{Forced choice}

We compared FCC accuracy at test across the five delay conditions (minimal interference 1, cognitive interference, feature-level visual interference, low-level visual, minimal interference 2) between NC and controls using Crawford's modified $t$-tests (Crawford and Howell 1998; Crawford et al. 2006) and $z_{\mathrm{cc}}$ effect sizes (Crawford et al. 2010). NC performed below controls on the cognitive interference $\left(t_{(10)}=2.06, P=0.03, z_{\mathrm{cc}}=2.14\right)$, feature-level visual interference $\left(t_{(10)}=2.64, P=0.01, Z_{\mathrm{cc}}=2.46\right)$, and low-level visual interference conditions $\left(t_{(10)}=4.69, P<0.01, Z_{\mathrm{cc}}=4.83\right)$. Critically, NC's performance was not significantly different from controls on either minimal interference condition $\left(t_{(10)} \mathrm{s}<1.31, P \mathrm{~s}>0.11\right.$, $Z_{\mathrm{CC} S}<1.52$ ) (Fig. 3A).

\section{Yes/No}

As described above, we compared performance on the YN task across all five conditions between NC and controls. We used signal detection theory to characterize our data, calculating a nonparametric discriminability measure $\left(A^{\prime}\right)$ from the proportion of hits and false alarms in the YN test (MacMillan and Creelman 2005). We used Crawford's modified $t$-tests to compare NC and control

\section{A Forced choice with corresponding foils (FCC)}
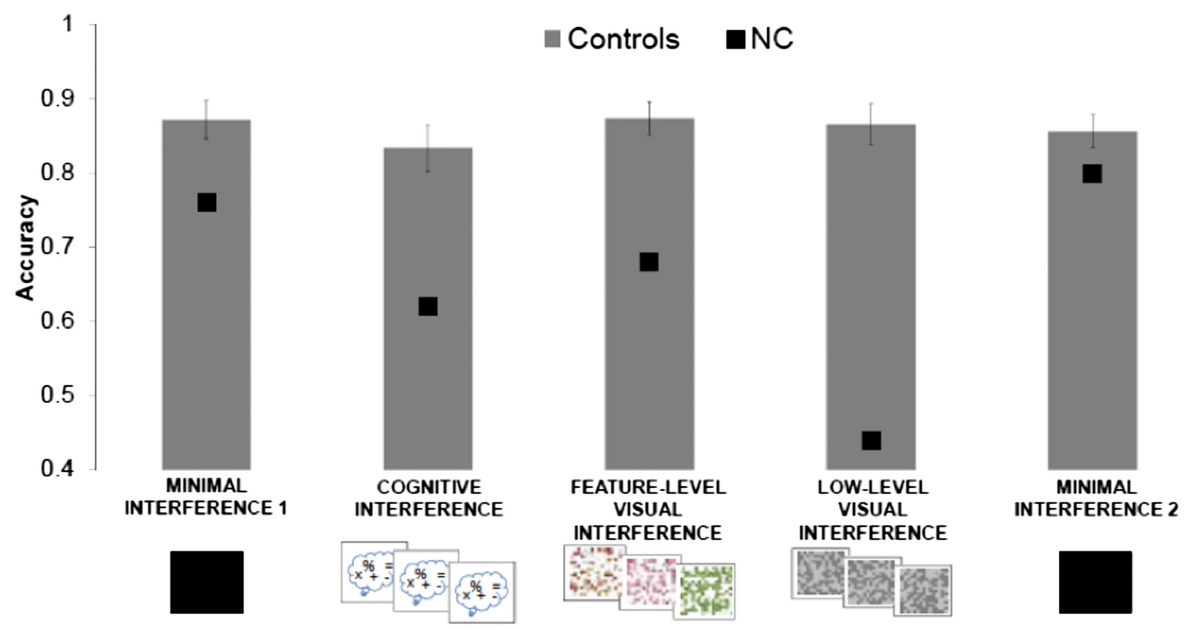

\section{B Yes/No (YN)}
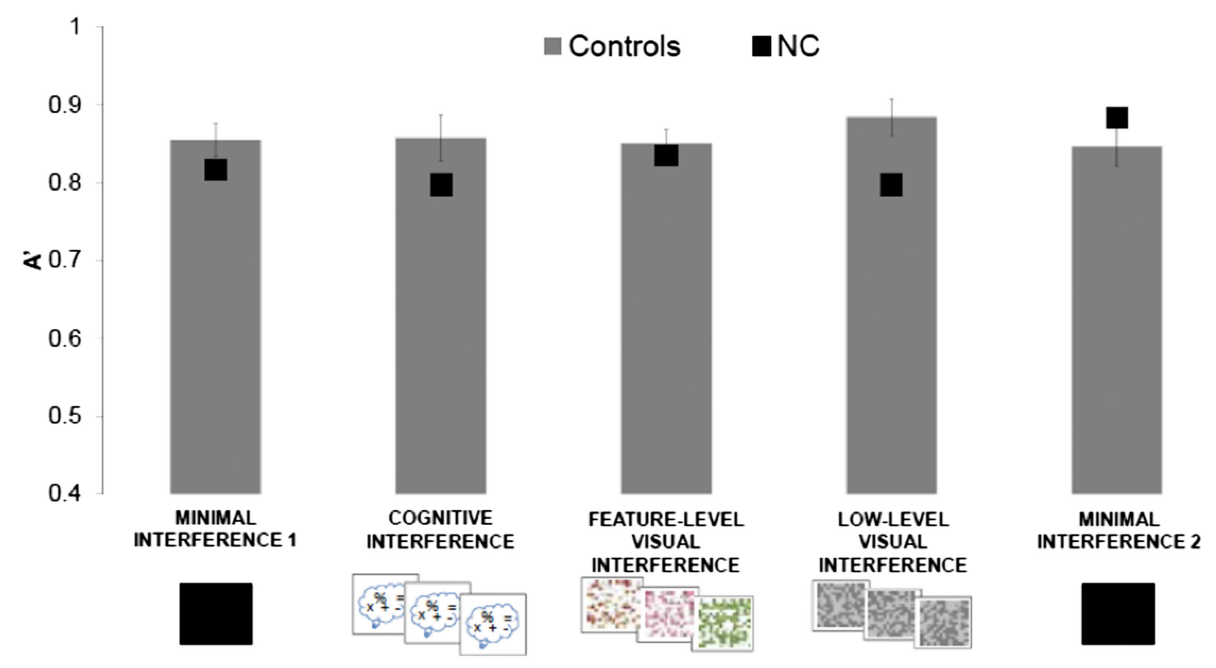

Figure 3. (A) FCC accuracy (percent correct). NC was significantly impaired on all high interference conditions, but did not significantly differ from control on either minimal interference condition. $(B) \mathrm{YN}$ accuracy $\left(A^{\prime}\right)$. N $C^{\prime}$ 's performance was not significantly different from that of controls in any condition on the YN task. A bootstrap resampling procedure revealed a significant three-way interaction whereby, compared to controls, NC was disproportionately impaired on all three high interference conditions in the FCC task relative to YN. Error bars indicate standard error of the mean. 
performance. NC's performance was not significantly different from controls in any condition (minimal $1, t_{(10)}=0.56, P=0.29$, $z_{\mathrm{cc}}=0.59$; cognitive, $t_{(10)}=0.62, P=0.27, z_{\mathrm{cc}}=0.65$; feature-level, $t_{(10)}=0.26, P=0.39, z_{\mathrm{cc}}=0.27$; low-level, $t_{(10)}=1.12, P=0.14, Z_{\mathrm{cc}}$ $=1.17$; minimal $\left.2, t_{(10)}=-0.43, P=0.33, z_{\mathrm{cc}}=-0.46\right)$ (Fig. $\left.3 \mathrm{~B}\right)$.

\section{Between-group interactions}

Next, we wished to directly examine whether test format (FCC versus $\mathrm{YN}$ ) differentially affected $\mathrm{NC}$ 's vulnerability to interference, relative to controls. That is, we aimed to test the three-way interaction between group, interference type, and test format. To accomplish this goal, we used a bootstrap resampling procedure as a means to estimate the distributions of performance between test formats. We then compared NC's performance to the resulting distributions (see Statistical Analyses section in the Materials and Methods for a detailed description of this procedure). For the omnibus three-way interaction analysis, we collapsed across both minimal interference conditions and our three high interference conditions to obtain difference scores between minimal and high interference within test format, for both the control group and patient NC. We then subtracted those difference scores across test format for both groups and compared NC's difference-ofdifferences score to the resulting distribution of controls' scores. The results show a significant three-way interaction whereby NC's vulnerability to interference (minimal versus high difference) was greater in the FCC task than in YN, relative to controls. That is, NC's performance fell outside the $95 \%$ confidence intervals of the control distribution, $(\mathrm{NC}=1.41,95 \%$ CI $[-0.82,0.84]$, $P<0.01$, Fig. 4A).

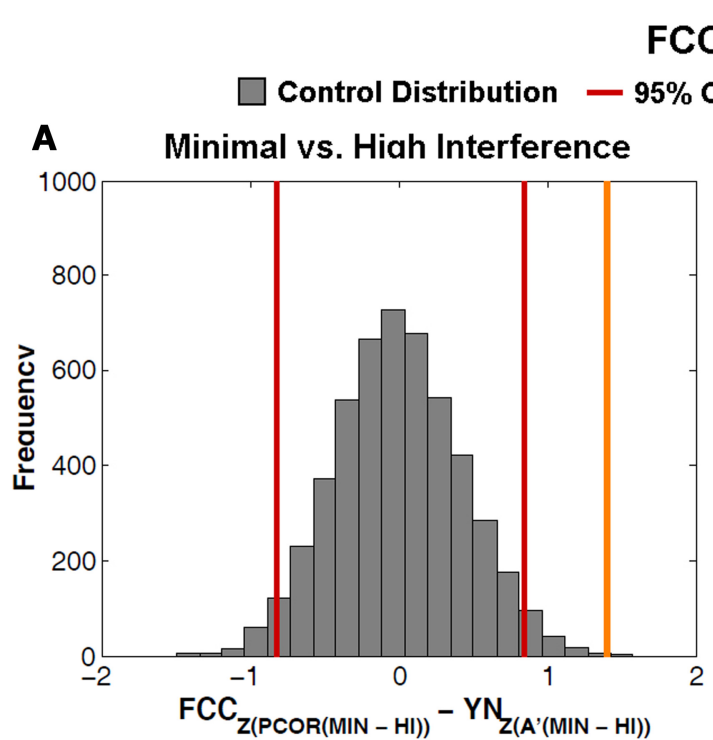

FCC vs. YN

A Minimal vs. Hiah Interference

B Minimal vs. Cognitive Interference

C Minimal vs. Feature-Level Interference
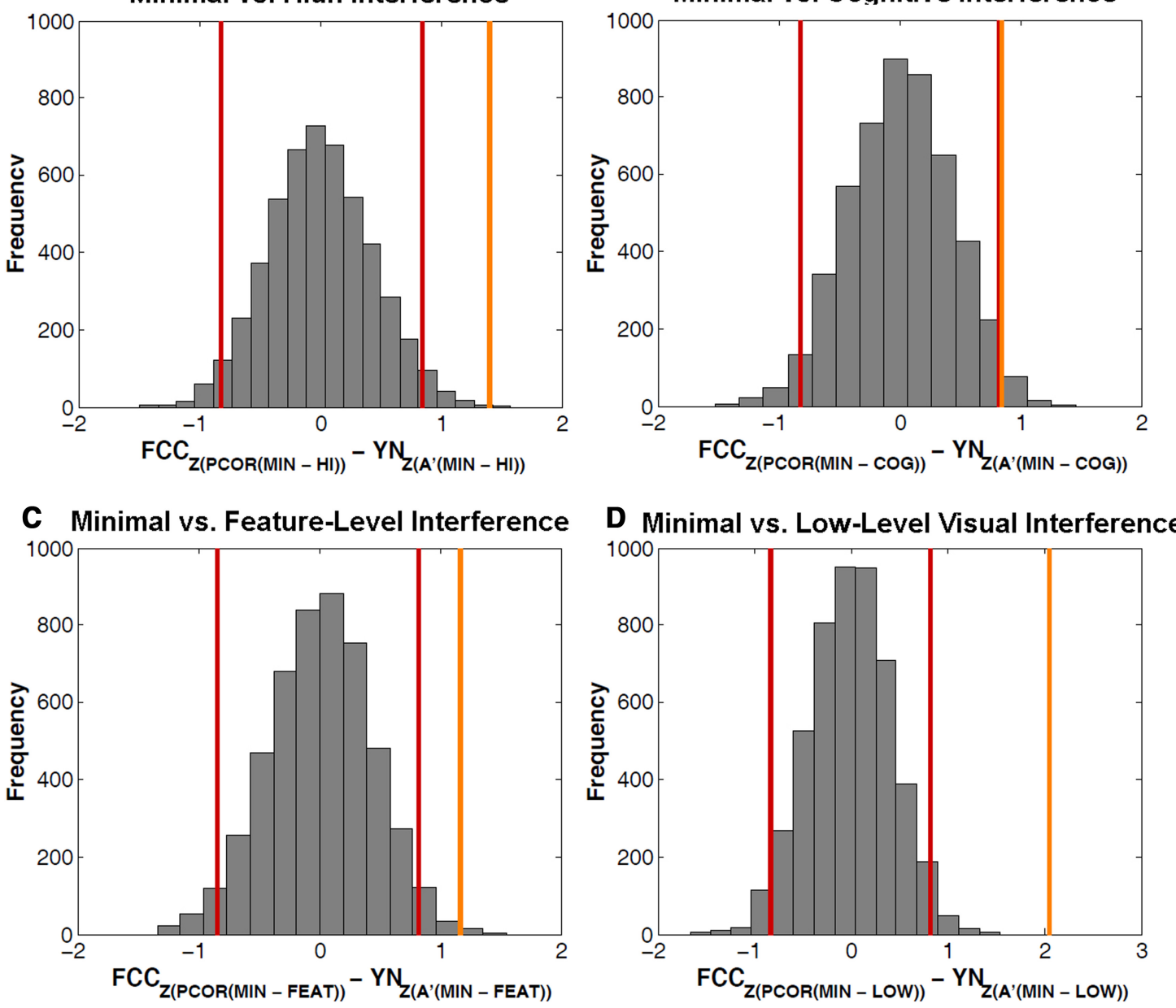

Figure 4. Distribution of difference-of-difference scores for all three-way interactions between Groups (NC versus controls) $\times$ test format (FCC versus YN) $\times$ Interference Types. We calculated within test format differences between $(A)$ Minimal Interference and High Interference (collapsed across both "minimal" and three "high" conditions; "MIN" versus "HI" on the $x$-axis). (B) Minimal interference conditions and cognitive interference ("MIN" and "COG" on the $x$-axis). (C) Minimal interference conditions and feature-level interference ("MIN" and "FEAT" on the $x$-axis). ( $D$ ) Minimal interference conditions and low-level visual interference ("MIN" and "LOW" on the $x$-axis). (A-D) Differences were taken for both controls and patient NC. We then compared the difference scores across tasks (FCC-YN) by subtracting each control subject's difference score in FCC with every other subject's in YN. We used proportion correct ("PCOR" on $x$-axis) for FCC and A' for YN. We then $z$-scored both DVs before calculating across-task differences. The gray bars represent the resulting distribution of mean difference-of-differences scores for controls. Red lines represent $95 \%$ confidence intervals. The orange line represents $\mathrm{NC}^{\prime}$ s difference-of-differences score. For all three interactions, NC's score falls outside the $95 \%$ interval of the control distribution, making all interactions significant (all $P^{\prime} s=0$ ). 
We then tested whether this interaction was driven by a single interference condition. We found that, compared to controls, NC's performance was impaired by each interference type to a greater extent in the FCC task than in the YN task (Fig. 4B-D). NC's difference-of-differences score fell outside the $95 \%$ confidence intervals for each contrast: minimal versus cognitive interference $(\mathrm{NC}=0.84,95 \% \mathrm{CI}[-0.83,0.82], P<0.05)$, minimal versus featurelevel $(\mathrm{NC}=1.16,95 \% \mathrm{CI}[-0.85,0.83], P<0.01)$, and minimal versus low-level $(\mathrm{NC}=2.05,95 \% \mathrm{CI}[-0.84,0.81], P=0)$. These analyses support the claim that $\mathrm{NC}$ was disproportionately impacted by each interference type in the FCC task to a greater degree than in the YN task, relative to controls. We note that all of the results reported above did not differ qualitatively when we calculated YN performance as $d^{\prime}$ or as proportion correct.

Last, we compared NC's performance to controls within the FCC condition to examine whether he was differentially impacted by a specific type of high interference (Fig. 5). While there was no significant interaction between cognitive and feature-level interference $(\mathrm{NC}=-0.06,95 \% \mathrm{CI}[-0.09,0.01], P=0.45)$, there was an interaction between cognitive and low-level interference $(\mathrm{NC}=0.18,95 \% \mathrm{CI}[-0.07,0.01], P=0)$ and between feature-level and low-level interference ( $\mathrm{NC}=0.24,95 \%$ CI [-0.05, 0.07], $P=$ $0)$. Thus, within the FCC task, NC was impacted to the greatest degree by low-level interference relative to both cognitive and feature-level interference when compared to the pattern of performance in controls. However, the finding that NC's performance was significantly impaired relative to minimal interference on all three conditions supports the overarching claim that NC was unable to resist interference from all three interference types in the FCC condition.

\section{Discussion}

The present study investigated recognition memory in a developmental amnesic person, NC, who presents with relatively selective damage to the MDN of the thalamus. This rare lesion profile provided a unique opportunity to test two hypotheses: (i) that MDN damage would lead to selective deficits in a task that relies on PRC output, and (ii) that these deficits would be sensitive to the presence of interference. To investigate these possibilities, we administered two object recognition memory tests with similar foils with different types of interference during the study-test delay: a YN test that requires the hippocampus, and a FCC test that is supported by PRC. Consistent with the prediction that the MDN is a critical output structure for PRC, we found that NC exhibited intact performance in the $\mathrm{YN}$ test across conditions, but was impaired in the FCC test. Critically, however, as predicted by the representational-hierarchical account, this FCC deficit emerged only under conditions of interference. These results provide the first demonstration that damage to the MDN leads to selective and interference-dependent memory impairments in a task that relies on PRC output. In doing so, they fill a key gap in the literature, providing evidence for dissociable contributions of the ATN and MDN to recognition memory.

The role of test format in recognition memory deficits The first aim of the present study was to test the prediction that damage to the MDN, proposed a key output structure of PRC, would lead to selective deficits in FCC recognition memory with similar foils. This prediction is in line with previous proposals that the hippocampus is critical for performance in the YN test format, whereas PRC can support performance in the FCC test format (Norman 2010). Consistent with this distinction, the present results identify deficits in FCC, but not YN, performance in a patient with MDN damage. These results not only lend critical support to the proposal that the neural mechanisms supporting recognition memory performance vary across test formats when targets and foils share overlapping features, but also provide novel evidence that such dissociations extend beyond the MTL to include diencephalic regions. Moreover, the present results demonstrate that the PRC-MDN system is not only sufficient, but necessary to support successful mnemonic discriminations in the FCC test format.

The contribution of the PRC-MDN pathway to FCC recognition has been characterized both in terms of the nature of the representations maintained in PRC, and in terms of the mnemonic processes that these brain regions support, and these alternative theoretical explanations are elaborated upon below. But according to both accounts, PRC contains item representations that are capable of supporting judgments of an item's prior occurrence based on stimulus familiarity (Cowell et al. 2006; Diana et al. 2007). In a FCC

\section{Comparisons Between Interference Types Within FCC $\square$ Control Distribution $-95 \%$ Confidence Intervals - NC's Score}
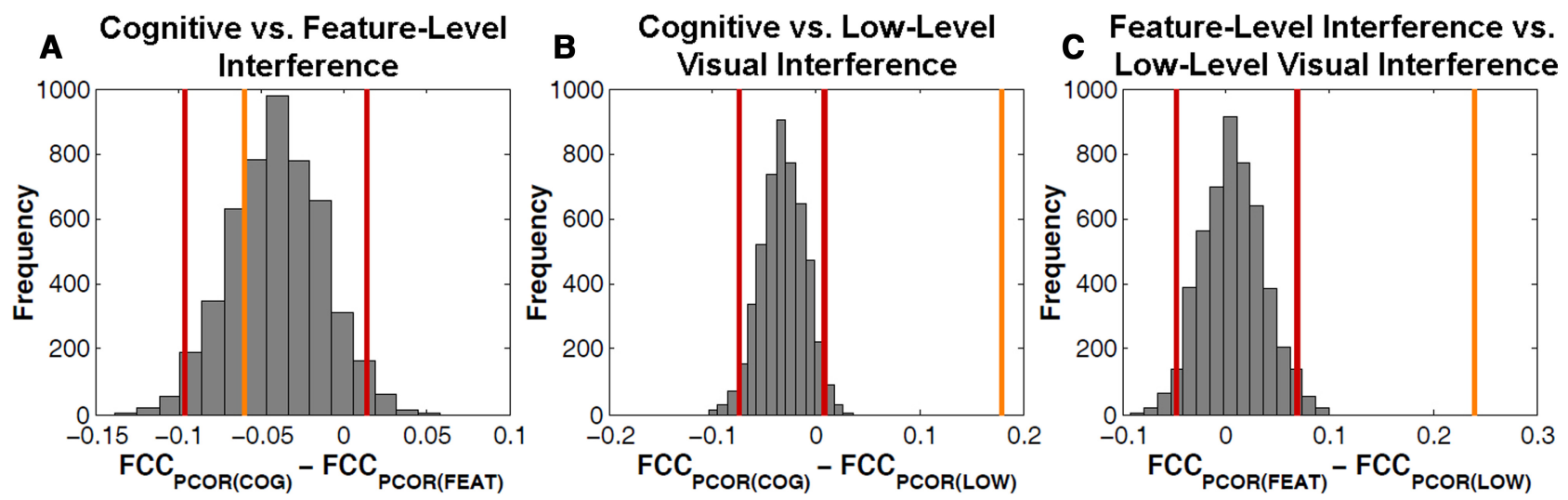
C Low-Level Visual Interference

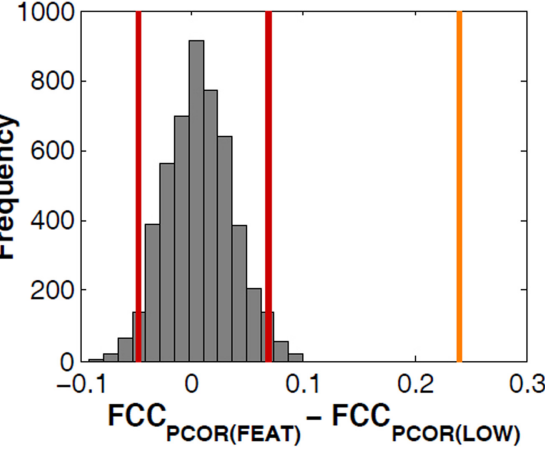

Figure 5. Distribution of difference scores within FCC between each high interference condition. We calculated difference scores between ( $A$ ) cognitive and feature-level visual interference, $(B)$ cognitive and low-level visual interference, $(C)$ feature-level visual and low-level visual interference. $(A-C)$ Differences were taken for each FCC participant and patient NC. Red lines represent $95 \%$ confidence intervals. The orange line represents NC's difference score. Where NC's score falls within the $95 \%$ interval, there is no significant difference $(P=0.45)$. 
test, in which targets and foils are presented simultaneously, such familiarity signals are said to reliably distinguish between targets and similar foils by enabling a comparison of the cortical memory strength elicited by each item (Norman 2010).

Although PRC is intact in NC, we propose that damage to the MDN impairs performance in the FCC object recognition memory test by reducing the ability for object-level representations supported by PRC to be relayed to the prefrontal cortex. This possibility is consistent with existing structural connectivity data, which suggests that the MDN serves as critical relay station between PRC and the prefrontal cortex (Amaral 1999; Saunders et al. 2005; Aggleton et al. 2011), as well as previous work demonstrating that MDN damage leads to a similar pattern of deficits as does disconnection of the prefrontal cortex and rhinal cortex (Parker and Gaffan 1998). In contrast, because PRC is intact in NC, object-level representations supported by this region can still reach the hippocampus (Fig. 1C), enabling the intact hippocampal-ATN system to support YN recognition memory.

Taken together, the observation that NC exhibited intact YN performance, coupled with impaired FCC performance, is consistent with the proposal of the MEMN model, which states that the ATN and MDN form parts of the extended hippocampal and PRC systems, respectively (Aggleton et al. 2011). Nevertheless, it should be noted that, in addition to the MDN lesion we have described so far, NC does have some visual abnormalities to the ATN, which may reflect an extension of the MDN lesion. As such, we cannot conclusively rule out the role of the ATN, but we think that it unlikely that abnormalities in the ATN are driving our empirical findings. First, the volume of the ATN was not significantly different than the volume of 10 age- and gendermatched controls; the only thalamic region with significantly reduced volume was the right MDN (Nasseri et al. 2017). Second, NC's performance on the YN task, which necessitates hippocampal outputs (Holdstock et al. 2002; Westerberg et al. 2013), was completely unaffected relative to controls across all interference conditions, suggesting that ATN function is not disrupted.

\section{Process-based versus representational accounts of recognition memory}

There exist a number of theoretical accounts of how the MTL underpins recognition memory. Some theories characterize the contribution of separate MTL structures in terms of computational or cognitive mnemonic processes such as pattern separation, recollection, and familiarity (Aggleton and Brown 1999; Norman and O'Reilly 2003; Norman 2010). These are process-based models in the sense that the computational or cognitive processes are hypothesized to depend on the MTL structure with which they are associated regardless of the stimulus material that is being encoded, stored, and retrieved. Other models instead emphasize representations alone, claiming that recognition memory is underpinned by whichever MTL structure possesses the most useful representations of the to-be-remembered information, regardless of whether pattern separation or judgment of familiarity is required by the task (Cowell et al. 2010; McTighe et al. 2010; Martin et al. 2011, 2013; O'Neil et al. 2013; Watson and Lee 2013; Yeung et al. 2013). A third class of models may be thought of as "hybrids," positing some combination of process-based and representational explanations, such as the notion that hippocampus is important for recollection but also for binding items to their context (Diana et al. 2007; Mayes et al. 2007; Ranganath 2010).

In fact, the predictions of process-based, hybrid and representational accounts for FCC versus YN are similar, despite the different underlying explanations outlined in the Introduction. That is, the accounts all predict that PRC damage should impair recognition memory in a FCC but not a YN test format, whereas hippocampal damage should produce the opposite pattern of impairment. However, only the representational-hierarchical account proposes an explicit mechanism by which visual interference should cause particular problems in the FCC format for an individual with compromised PRC representations (Cowell et al. 2006). For the other classes of theory, while such a finding would not necessarily be incompatible with their account, they neither explicitly predict nor offer a mechanistic explanation for this outcome.

An additional unique prediction of representational accounts, not tested in the present study, is that they do not predict that focal damage to PRC would elicit the same pattern of intact YN and impaired FCC performance observed here in patient NC. Instead, focal damage to PRC would be expected to reduce the availability of object-level representations to both YN and FCC performance; that is, if the item-level representations that distinguish distinct but similar objects cannot be uniquely bound with context in the hippocampus, this would result in impaired performance in the YN format as well as the FCC format. Although the current data cannot speak to this prediction, as PRC is volumetrically intact in NC, it represents an important testable hypothesis that should be explored by future research.

\section{The role of interference in recognition memory deficits}

A key, unique prediction of the representational-hierarchical account for the present study was that recognition memory deficits in the FCC test, if observed, would not be all or none, but rather dependent on the presence of interference. This prediction arises because memory impairment following MTL damage is proposed to be driven by increased vulnerability to interference (Cowell et al. 2006; McTighe et al. 2010; Barense et al. 2012). Specifically, this view states that damage to PRC reduces the availability of object-level representations that are critical for disambiguating objects with visually similar features, thus increasing reliance on more simple, feature-level representations that are more vulnerable to interference from exposure to visual features. Accordingly, deficits in performance following damage to the PRC system should not be all or none, but rather dependent on the presence of interference. In the present study, we tested the prediction that damage to the MDN, a key output structure for PRC, would also cause a selective vulnerability to interference on tasks that rely directly on PRC output, such as the FCC test in the current study. Consistent with this prediction, NC exhibited interferencedependent deficits in FCC performance, whereas YN performance remained intact across all conditions.

In particular, NC exhibited impaired FCC performance when the study-test delay was filled with interference, but intact performance in the minimal interference condition, during which he sat with eyes closed in a darkened room throughout the study-test delay. Of note, intact FCC performance was observed both when the minimal interference condition was presented at the beginning of the experiment (minimal interference 1), and when it was presented last (minimal interference 2), suggesting that NC's performance could still be rescued after the high interference conditions presented in the middle of the experiment. The observation that minimizing delay-period interference enhanced performance on a PRC-dependent task adds to a growing body of work identifying benefits of reducing interference on memory and perception in humans and rodents with compromised PRC integrity (Cowan et al. 2004; Della Sala et al. 2005; McTighe et al. 2010; Barense et al. 2012; Newsome et al. 2012; Romberg et al. 2012; Monti et al. 2014). Critically, the present results extend this work by identifying an analogous effect following selective MDN damage, lending support to the proposal that damage to a key output structure in the PRC pathway can also lead to increased 
vulnerability to interference, by virtue of disconnecting information output from the PRC.

Interestingly, the effects of interference on FCC performance were not limited to the feature-level visual interference condition, which was predicted based on prior work in humans (Barense et al. 2012) and rodents (McTighe et al. 2010) with PRC lesions. Instead, $\mathrm{NC}$ was impaired relative to controls across all three interference conditions, with the magnitude of this impairment significantly greater following the low-level visual interference condition relative to the cognitive interference and feature-level visual interference conditions, which did not differ from one another (Fig. 5). We are not aware of any theoretical framework that would predict the low-level condition to produce the greatest impairments. On the one hand, this pattern may suggest that any form of active interference during the study-test delay is sufficient to disrupt performance following MDN damage. For instance, it has been suggested that engagement in any cognitive activity during the delay period triggers the formation of new memories for these events, thus impairing consolidation of the recently learned material (Wixted 2004; Dewar et al. 2007). Although we cannot rule out this possibility, we think it unlikely that this can fully explain the observed pattern of results. In particular, if delay-period interference impaired memory performance by interrupting consolidation processes, one would expect the formation of new memories to be impaired across test formats; instead, we observed that YN performance was unaffected. Furthermore, the observation that costs to performance were not equivalent across conditions, but greater in the low-level visual interference condition, suggests that the nature of delay-period interference is also relevant to the mechanisms that disrupt performance.

An alternative possibility is that the low-level visual interference condition and cognitive interference conditions inadvertently created exposure to relevant feature-level interference, despite our intentions to minimize such exposure in these conditions. For example, during the low-level visual interference delay condition, $\mathrm{NC}$ asked the experimenter (RNN) whether faces were hidden in the scrambled gray images. Although this was not the case, the illusory perception of face stimuli in the presence of white noise would nevertheless be associated with analogous activation of ventral occipitotemporal cortex as perceptual face processing, as described in previous work (Smith et al. 2012). Thus, it is possible that this condition exposed NC to a more complex level of interference than intended. Similarly, although the cognitive interference condition involved doing arithmetic problems with eyes closed, we cannot rule out the possibility that $\mathrm{NC}$ used visual imagery to solve the problems, which may have also introduced unforeseen visual interference. Importantly, it is possible that even low-level visual information, such as the edges and shapes that were present within the scrambled grayscale images in the low-level visual interference condition, was sufficient to produce interference that was detrimental to performance. That is, in the absence of intact PRC output, it is unclear what visual features are available to support performance, thus making it difficult to predict the exact type of visual features that will lead to maximally detrimental interference. Accordingly, even low-level visual information may have been sufficient to produce interference that affected performance in the FCC test format.

Finally, it is possible that MDN damage leads to increased vulnerability to interference in a manner that is similar, but not identical to that following PRC damage. Indeed, previous work comparing MDN and PRC lesions in nonhuman primates has provided preliminary evidence for at least some differences in the profiles of performance observed across lesion profiles (Parker et al. 1997). An additional factor to consider in the interpretation of these results is the developmental nature of NC's lesion. NC experienced a stroke only a few days after birth, at a time when the brain is incredibly plastic and reorganization can occur (Vargha-Khadem et al. 2003). Very little is known about the effects of early thalamic damage on cognitive development, yet we note that NC does show similarities to other developmental amnesic cases, including impaired episodic memory with relatively spared semantic memory (Vargha Khadem et al. 1997; D'Angelo et al. 2015, 2016; Ryan et al. 2015). It is possible that the perinatal timing of the lesion differentially affected both thalamic development as well as recognition memory. Future research in patients with selective MDN lesions will be necessary to determine the specificity of interference effects following MDN damage, including the degree to which they are driven by low-level features, complex conjunctions of features, or simply any active interference task that occurs between study and test.

Although we cannot draw definitive conclusions regarding the exact mechanisms underlying the effects of interference on FCC performance, the present results significantly advance our understanding of the role of the MDN in recognition memory. In particular, the present data provide novel evidence for selective and interference-dependent deficits in FCC object recognition memory with similar foils following damage to the MDN of the thalamus. These results are consistent with a selective role of the MDN in supporting performance on tasks that rely directly on PRC output, as proposed by the MEMN model. This model states that different thalamic nuclei, the MDN and ATN, make distinct contributions to PRC- and hippocampal-dependent memory, respectively. Although this distinction is often couched in terms of different processes supported by these two systems (i.e., familiarity and recollection), here we suggest that this dissociation may be best understood in terms of the representations supported by these two systems, as outlined by the representational hierarchical framework. Critically, such an account can explain both the distinct contributions of the PRC and hippocampal systems across test formats when targets and foils are perceptually similar, as well as observations that memory deficits following damage to these systems are modulated by interference. Collectively, the present results advance our understanding of the joint contributions of the MTL and thalamic nuclei to recognition memory, and highlight the value of a representational framework for understanding the deficits that emerge when these regions are compromised.

\section{Materials and Methods}

\section{Participants}

NC was 21 yr old at the time of testing, and had completed high school and two years of technical college. As described previously (D'Angelo et al. 2015), NC experienced a thalamic stroke days after birth. This damage is greater in the right than left hemisphere, and primarily affected the MDN of the thalamus (Fig. 6). Upon visual inspection, a radiologist noted abnormalities to the anterior nucleus, which may have been an extension of the MDN lesion. However, there is no appreciable volume loss within this region. That is, detailed volumetric analysis showed a significantly smaller right MDN and numerically smaller left MDN. In contrast, the volume of the ATN was not different from controls-in fact, it was numerically larger (Nasseri et al. 2017). NC's hippocampus and MTL are intact and not significantly different in subregion volume than that in controls. Previous reports have indicated that NC's fornix integrity may also be compromised (D'Angelo et al. 2015). However subsequent qualitative assessment of NC's fornix indicates that although his fornix may be reduced in volume, it is neither severed nor does it show gross abnormalities based on qualitative analysis of DTI data (quantitative DTI analyses are currently underway). Although we cannot rule out involvement of the fornix, fornix integrity would be critical for YN recognition tasks with similar foils, as they require intact input/output from the hippocampus, rather than $\mathrm{YN}$ recognition without similar foils, which 


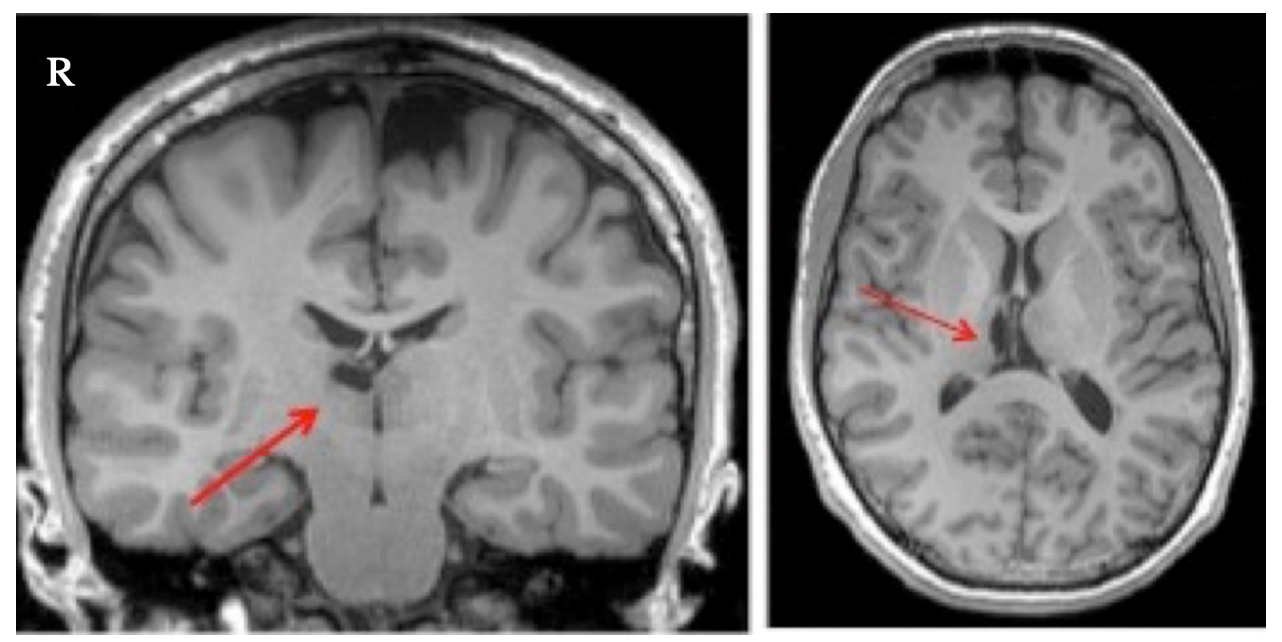

Figure 6. T1-weighted MRI scans of NC with arrow denoting infarct in the right MDN thalamus, with significant volume loss.

could be supported by PRC (Aggleton and Brown 1999; Brown and Aggleton 2001; Rudebeck et al. 2009; Aggleton et al. 2010, 2011; Bennett et al. 2014; Bennett and Stark 2015). NC's intact performance on the current YN task is inconsistent with fornix damage.

A detailed neuropsychological exam performed approximately $1 \mathrm{yr}$ prior to our experimental testing has been published previously and a summary is provided in Table 1 (D'Angelo et al. 2015, 2016). In summary, NC shows normal IQ function with slight impairments in working memory and greater impairments on episodic memory recall and recognition tests. NC was also borderline impaired on the Benton Facial Recognition test, which involves recognizing faces, and the more complex visuospatial processing in the Rey-Osterreith complex figure immediate recall. NC showed intact performance across measures of executive function, semantic knowledge, language and processing speed.

Two separate groups of young adult controls were recruited from the local Toronto community for each of the YN and FCC experimental tasks. Ten controls $\left(M_{\mathrm{age}}=20.3 \mathrm{yr}, \mathrm{SD}=1.63\right)$ were recruited for the FCC task and 10 controls $\left(M_{\mathrm{age}}=20.3 \mathrm{yr}, \mathrm{SD}=1.95\right)$ were recruited for the $\mathrm{YN}$ task. Both groups were matched in age with NC (Crawford's $t$ 's $>1$ ). All participants provided informed consent and were compensated for their time. The study was approved by the Ethics Review Boards of Baycrest Hospital and The University of Toronto.

\section{Experimental design}

Participants were administered an object recognition task with three phases (study, delay, test), five delay conditions (minimal interference 1 , cognitive interference, feature-level visual interference, low-level visual interference, minimal interference 2 ), and one of two test formats (FCC, YN) (Fig. 2).

\section{Study phase}

The first phase was an incidental encoding phase during which participants studied a single object on screen (target object) with the question "Is this object bigger than a shoebox?" Participants responded with one of two labeled keys on the keyboard to make their decision. Each condition (minimal 1, cognitive, featurelevel, low-level, minimal 2) consisted of 50 trials, each with unique stimuli. Each image was contained within an invisible box of $250 \times 250$ pixels in the center of the screen. Each stimulus remained on screen for $5 \mathrm{sec}$, regardless of when participants made their response so that an equal time was spent encoding each stimulus.

\section{Delay phase}

The second phase was a 10-min delay period, which varied across the different interference conditions. The delay period was always $10 \mathrm{~min}$, although participants were not explicitly aware of the time. For all participants, the first condition was always minimal interference 1, during which time we asked participants to sit quietly in a darkened room with their eyes closed. The experimenter remained in the room to ensure that participants did not sleep, talk, or otherwise engage in activities. We described the minimal interference phase to participants as a "brief rest." We administered the minimal interference 1 condition first to gain an estimate of baseline recognition memory performance prior to administration of highly interfering delay periods. We administered a second minimal interference condition at the end of the experiment (minimal interference 2) to both combat effects of test fatigue, and also to investigate whether having a final low interference condition could rescue any deficits seen in the higher interference conditions.

We consider the remaining three delay conditions to be our high interference conditions (cognitive, feature-level visual, lowlevel visual). The order of these high interference conditions was counterbalanced such that each condition was presented as the second, third, or fourth condition an equal number of times across participants. NC was administered the following counterbalances: FCC: minimal 1, cognitive, feature-level visual, low-level visual, minimal 2; YN: minimal 1, feature-level visual, low-level visual, cognitive, minimal 2 . We designed the cognitive interference condition to investigate the hypothesis that impaired consolidation contributes to impaired performance following delay-period interference (Wixted 2004). To that end, we filled the delay period with mental effort but minimal visual input. During the cognitive interference condition, we asked participants to again close their eyes, and then asked them to perform verbal mental arithmetic questions. We attempted to ask questions that would minimize visual imagination as much as possible. The cognitive questionnaire included items such as "Subtract 7 from 100 and keep subtracting by 7 until I tell you to stop," "Today is Tuesday, August 16. What will the date be on next Wednesday?" and "What is $75 \%$ of 16 ?" Participants were told the task was meant to exert mental effort, and that we did not expect them to perform perfectly. The experimenter kept track of the time it took to administer the cognitive questionnaire, and stopped when the $10 \mathrm{~min}$ was filled, regardless of the number of items completed.

We designed the feature-level visual interference condition to investigate our hypothesis regarding the representationalhierarchical model (Cowell et al. 2006). That is, we predicted that with highly similar visual stimuli, interference from low-level features of the foil objects would interfere with correct recognition of 
Table 1. Neuropsychological profile of NC, data from D'Angelo et al. $(2015,2016)$.

\begin{tabular}{|c|c|}
\hline Test & Normed score \\
\hline \multicolumn{2}{|l|}{ General intelligence } \\
\hline WAIS-IV: full scale IQ (standard score) ${ }^{\mathrm{a}}$ & 94 \\
\hline Verbal comprehension index & 101 \\
\hline Perceptual reasoning index & 106 \\
\hline Working memory index & 76 \\
\hline Processing speed index & 91 \\
\hline \multicolumn{2}{|l|}{ Semantic knowledge } \\
\hline WAIS-IV vocabulary (scaled score) ${ }^{a}$ & 10 \\
\hline \multicolumn{2}{|l|}{ Language production } \\
\hline Boston naming test (percentile) ${ }^{\mathrm{b}}$ & 39th percentile \\
\hline Semantic fluency (animals) $(z \text {-score })^{c}$ & 1.47 \\
\hline \multicolumn{2}{|l|}{ Anterograde memory } \\
\hline \multicolumn{2}{|l|}{ WMS-IV logical memory } \\
\hline Logical memory I: immediate recall (scaled score) & 7 \\
\hline Logical memory II: delayed recall (scaled score) & 2 \\
\hline Logical memory II: recognition (percentile) & 3-9th percentile \\
\hline \multicolumn{2}{|l|}{ California verbal learning Test-II } \\
\hline Total trials 1-5 ( $t$ score) & 29 \\
\hline Short delay free recall ( $z$-score) & -2.5 \\
\hline Short delay cued recall ( $z$-score) & -1.5 \\
\hline Long delay free recall ( $z$-score) & -2.5 \\
\hline Long delay cued recall ( $z$-score) & -3 \\
\hline Learning ( $z$-score) & -1.5 \\
\hline Total intrusions $(z \text {-score })^{\mathrm{d}}$ & 5 \\
\hline Total repetitions $(z \text {-score })^{d}$ & 1.5 \\
\hline Recognition (hits) (z-score) & 0.5 \\
\hline Recognition (false positives) ( $z$-score) & 3 \\
\hline Discrimination & -1.5 \\
\hline \multicolumn{2}{|l|}{ Rey-Osterrieth complex figure ( $t$ score) } \\
\hline Immediate recall & $<20$ \\
\hline Delayed recall & $<20$ \\
\hline \multicolumn{2}{|l|}{ Processing speed } \\
\hline WASI-IV coding ${ }^{\mathrm{a}}$ & 7 \\
\hline WASI-IV symbol search ${ }^{a}$ & 10 \\
\hline \multicolumn{2}{|l|}{ Visuospatial function } \\
\hline WAIS-IV block design ${ }^{\mathrm{a}}$ & 13 \\
\hline Rey-Osterrieth complex design-copy (percentile) & 11-16th \%ile \\
\hline Judgment of line orientation (percentile) & 72nd \%ile \\
\hline Benton facial recognition test & Borderline \\
\hline \multicolumn{2}{|l|}{ Working memory } \\
\hline WAIS-IV letter-number sequencing ${ }^{a}$ & 6 \\
\hline WAIS-IV digit span ${ }^{\mathrm{a}}$ & 5 \\
\hline \multicolumn{2}{|l|}{ Attention and executive function } \\
\hline \multicolumn{2}{|l|}{ Trail making test $(z \text {-score })^{\mathrm{e}}$} \\
\hline Part A (sec) & -0.74 \\
\hline Part B (sec) & -0.95 \\
\hline Phonemic fluency (FAS) $(z \text {-score })^{c}$ & 0.31 \\
\hline WAIS-IV similarities (scaled score) ${ }^{\mathrm{a}}$ & 10 \\
\hline WAIS-IV matrix reasoning (scaled score) ${ }^{\mathrm{a}}$ & 11 \\
\hline
\end{tabular}

WAIS-IV, Wechsler Adult Intelligence Scale-IV; WMS-IV, Wechsler Memory Scale-IV.

${ }^{a}$ Canadian norms.

b In house norms.

'Tombaugh et al. (1999).

${ }^{\mathrm{d}}$ Lower scores indicate better performance.

${ }^{\mathrm{e}}$ Tombaugh (2004).

the high-level conjunctive representation of the target object. To that end, we presented participants with scrambled images of the foil objects during a passive viewing task. We created the scrambled images using a $20 \times 20$ pixel filter in Adobe Photoshop CS3 (Adobe Systems Incorporated). This pixel size allowed low-level features to be visible (i.e., color, line segments), but was not big enough to identify the object. Critically, the target and foil objects were very similar, so some of these low-level features were shared across the target and foil objects. Participants viewed each scrambled image for $11.5 \mathrm{sec}$, comprising a total of $10 \mathrm{~min}$ of the delay period. Participants were instructed to watch the screen as if they were watching television.
Last, we designed the low-level visual interference condition to serve as a visual control for the feature-level visual interference condition. We wanted to present low-level visual stimuli while eliminating the presence of the objects' lower-level visual features. As such, we developed stimuli similar to traditional white noise with scrambled dark and light gray squares. These stimuli were subjected to the same $20 \times 20$ pixel block filter as described above (Fig. 2). Participants viewed each gray image scramble for $11.5 \mathrm{sec}$, comprising a total of $10 \mathrm{~min}$ of the delay period. Participants were instructed to watch the screen as if they were watching television.

\section{Test phase}

Following the delay period, participants were either administered the FCC test or the YN test, and within a given testing session, the test format did not change. NC completed both the FCC and YN tests, with the FCC task completed approximately 1 wk prior to the YN task to reduce the likelihood of order effects on performance. Unique stimuli were used in the FCC and YN test. In the FCC test (Fig. 2), both the target and perceptually similar foil objects were displayed simultaneously, side-by-side. Participants were asked to indicate via labeled keyboard button press which object (left or right) they had seen previously in the study phase. Correct responses were counterbalanced such that either a left or a right response was equally likely.

In the YN test (Fig. 2), either the target or foil object was displayed on screen. Participants were asked to indicate whether they had seen the object previously in the study phase (yes or no). Half of the trials consisted of an old, previously seen target stimulus, and half of the trials consisted of a new foil stimulus that was perceptually similar to one of the targets in the preceding study phase. All conditions were counterbalanced such that a target stimulus for one participant would occur as a foil stimulus for another participant. A stimulus only appeared within its study/ test condition, and never in a different condition (i.e., if the green pillow in the example in Figure 2 appeared in the cognitive interference condition, it would never appear in any other condition for that participant).

In the test phase, participants had an unlimited amount of time to respond, and the experiment moved forward onto the next trial after a response was indicated. Including time for informed consent, a brief health demographics questionnaire, and debriefing, the entire experiment took between 1.5-2 $\mathrm{h}$ to complete.

\section{Stimulus validation}

We used unique stimuli across the FCC and YN tasks. Using Amazon Mechanical Turk (https://www.mturk.com), we asked a separate group of 16 participants to estimate the visual similarity of the target and foil objects. All participants were from the United States, gave informed consent, and were paid $\$ 9$ for $\sim 30$ $45 \mathrm{~min}$ of their time.

We picked equivalently rated objects for FCC and YN tasks, respectively $(t s<1)$. We intentionally removed highly similar semantic and perceptual objects from within a stimulus set to reduce within-experiment interference (i.e., high heels would not show up in the same condition as ballet flats; a donut-shaped pool float would not show up in the same condition as a bagel). Of course it is nearly impossible to have 250 unique stimulus pairs per experiment type without any perceptual or semantic overlap, which could increase interference across the experiment. For example, items from a single category, such as sports, or items found in a kitchen, would have semantic relationships with one another. Other items may overlap in color or texture and thus have perceptual relationships with one another.

\section{Statistical analyses}

We first compared NC's performance to controls within each condition using Crawford's modified $t$-tests (Crawford and Howell 1998; Crawford et al. 2006) and $z_{\mathrm{cc}}$ effect sizes (Crawford et al. 2010). 
Next, we directly tested whether NC's vulnerability to interference was specific to either test format (FCC versus YN) relative to controls. Specifically, we aimed to test the three-way interaction between group, interference type, and test format. To accomplish this, we used a bootstrap resampling procedure as a means to estimate the distributions of performance between test formats. We then compared NC's performance to the resulting distributions. Previous research has established bootstrapping as a viable method to compare patients with control groups (Parker 2006; Garrido et al. 2008; Busigny et al. 2014).

To test the omnibus three-way interaction, we collapsed across the two minimal interference conditions (minimal 1 and minimal 2) and the three high interference conditions (cognitive, feature-level, and low-level) within each test format, for all participants and for NC. We then calculated a within-test format difference score for each participant by subtracting high interference from minimal interference. To make our dependent variables comparable across test formats (i.e., proportion correct is used for FCC and A' for YN), we computed $z$-scores of the difference scores. Last, we calculated the difference between each participant's FCC $z$-scored difference score and every other participant's YN $z$-scored difference score. Because we had 10 participants for each test format, we obtained 100 between-participant difference-of-differences scores. Specifically, we subtracted participant one's FCC difference score from all $10 \mathrm{YN}$ subject's difference scores, then repeated this process for participant two's FCC difference score, and so on, to obtain 100 between-participant scores. Last, we estimated the distribution of mean difference-of-differences for control participants by running 5000 bootstrap resamples and calculating 95 percentile confidence intervals (CI) for the resulting distribution to assess its reliability (Efron 1992). Specifically, because our across-task comparison resulted in 100 difference scores, we resampled 10 scores with replacement, took the mean of the subset, repeated 5000 times, and plotted the resulting distribution of 5000 means. For a within-subject bootstrapping analysis it is standard procedure to resample all scores. However, because our large pool of 100 difference scores resulted from between-subject comparisons, resampling all 100 scores would create a distribution essentially representing 100 subjects on a within-subject task. Thus, to introduce additional variance contributed by the between-subject nature of the design, we resampled the number of scores we would have expected had the test format manipulation been within-subject.

Next, we tested whether the omnibus three-way interaction was driven by a single interference condition. We reran the above three-way analysis by first calculating separate within-test format difference scores for minimal and cognitive, minimal and featurelevel visual, and minimal and low-level visual interference. We then repeated all of these above procedures to compare differenceof-differences scores across test formats.

Last, we examined performance between each of the high interference types (cognitive, feature-level, and low-level) within the FCC task. We were motivated to examine differences between interference conditions due to NC's numerically poorer performance on the Low-Level Interference in the FCC task. To assess this, we calculated difference scores between each of the three interference conditions (cognitive versus feature-level, cognitive versus low-level, and feature-level versus low-level) within FCC for each participant. We then ran 5000 bootstrap resamples to estimate the distribution of mean difference scores and compared NC's difference score to the resulting distributions.

For all bootstrapped analyses, we calculated a discrete $P$-value by taking the proportion of bootstrap resamples that fell outside NC's difference-of-differences score as an approximation of the more commonly reported parametric $P$-value. It should be noted that unlike their parametric counterparts, discrete $P$-values can take on values of exactly 0 or 1 as they are not based on an assumed continuous distribution, but rather a discrete frequency distribution of bootstrap resamples. Additionally, unlike parametric CIs, percentile bootstrap CIs can be asymmetrical in shape, as they are calculated from empirical estimates of the distribution of the statistic, rather than an assumed distribution (Preacher and Hayes 2008). Our calculation of percentile CIs and discrete $P$-values is identical to the implementation in the "mediation" package for R (Tingley et al. 2014) and as reported elsewhere (Sun et al. 2017).

For each across-task comparison, it must be noted that we are comparing a distribution of mean differences that was calculated between-subjects to a single within-subject score (because we had two separate sets of control participants). Though this comparison is not ideal, we suggest that the distribution of mean difference scores between-subjects is likely more variable than a within-subject distribution. Specifically, a general principle favoring within-subject designs is that they allow for between-subject variance to be accounted for by an additional error term. In the current set of analyses, we were not able to account for the extra source of error. Additionally, it is common in the field of psychology for between-subject differences to be greater than differences accounted for by the experimental manipulation. Thus, we suggest that being unable to account for that variance here leads to a greater risk of committing Type II error than Type I error. Despite the fact that comparing NC's within-subject difference to a betweensubject distribution is likely a less powerful means of comparing test formats, we still find large differences between NC's performance and the between-subject distribution, suggesting that the deficits in NC's performance are indeed robust.

\section{Acknowledgments}

This work was supported by a Scholar Award from the James S McDonnell Foundation and an NSERC Discovery grant and Accelerator supplement to M.D.B. and NSERC grant 2015-04238 to R.S.R. M.D.B. and J.D.R. are also supported by the Canada Research Chairs program. We additionally thank collaborators involved in the MRI image acquisition, interpretation, and volumetric analyses referenced in our methods and shown in Figure 6: Mahsa Nasseri, Rosanna K. Olsen, Fuqiang Gao, Kie Honjo, Sandra Black, and Chris B. Martin. We thank NC and his family for their participation and all participants for their involvement.

\section{References}

Aggleton JP, Brown MW. 1999. Episodic memory, amnesia, and the hippocampal-anterior thalamic axis. Behav Brain Sci 22: 425-589.

Aggleton JP, Desimone R, Mishkin M. 1986. The origin, course, and termination of the hippocampothalamic projection in the macaque. J Comp Neurol 243: 409-421.

Aggleton JP, O'Mara SM, Vann SD, Wright NF, Tsanov M, Erichsen JT. 2010. Hippocampal-anterior thalamic pathways for memory: uncovering a network of direct and indirect actions. Eur J Neurosci 31: 2292-2307.

Aggleton JP, Dumont JR, Warburton EC. 2011. Unraveling the contributions of the diencephalon to recognition memory: a review. Learn Mem 18: 384-400.

Amaral DG. 1999. Introduction: what is where in the medial temporal lobes? Hippocampus 9: 1-6.

Barense MD, Groen I, Lee AC, Yeung LK, Brady SM, Gregori M, Kapur N, Bussey TJ, Saksida LM, Henson RN. 2012. Intact memory for irrelevant information impairs perception in amnesia. Neuron 75: 157-167.

Bartko SJ, Cowell RA, Winters BD, Bussey TJ, Saksida LM. 2010. Heightened susceptibility to interference in an animl model of amnesia: impairment in encoding, storage, retrieval-or all three? Neuropsychologia 48: 2987-2997.

Bennett IJ, Stark CE. 2015. Mnemonic discrimination relates to perforant path integrity: an ultra-high resolution diffusion tensor imaging study. Neurobiol Learn Mem 129: 107-112.

Bennett IJ, Huffman DJ, Stark CE. 2014. Limbic tract integrity contributes to pattern separation performance across the lifespan. Cereb Cortex 25: 2988-2999.

Brown MW, Aggleton JP. 2001. Recognition memory: what are the roles of the perirhinal cortex and hippocampus. Nat Rev Neurosci 2: 51-61.

Burke SN, Barnes CA. 2010. Senescent synapses and hippocampal circuit dynamics. Trends Neurosci 33: 153-161.

Burke SN, Wallace J, Hartzell A, Nematollahi S, Plange K, Barnes CA. 2011. Age-associated deficits in pattern separation functions of the perirhinal cortex: a cross-species consensus. Behav Neurosci 125: 836-847.

Busigny T, Van Belle G, Jemel B, Hosein A, Joubert S, Rossion B. 2014. Face-specific impairment in holistic perception following focal lesion of the right anterior temporal lobe. Neuropsychologia 56: 312-333. 
Bussey TJ, Saksida LM. 2007. Memory, perception, and the ventral visual-perirhinal-hippocampal stream: thinking outside of the boxes. Hippocampus 17: 898-908.

Carlesimo GA, Serra L, Fadda L, Cherubini A, Bozzali M, Caltagirone C. 2007. Bilateral damage to the mammillo-thalamic tract impairs recollection but not familiarity in the recognition process: a single case investigation. Neuropsychologia 45: 2467-2479.

Cowan N, Beschin N, Della Sala S. 2004. Verbal recall in amnesiacs under conditions of diminished retroactive interference. Brain 127: 825-834.

Cowan N, Beschin N, Perini M, Della Sala S. 2005. Just lying there, remembering: improving recall of prose in amnesic patients with mild cognitive impairment by minimising interference. Memory 13: $435-440$.

Cowell RA, Bussey TJ, Saksida LM. 2006. Why does brain damage impair memory? A connectionist model of object recognition memory in perirhinal cortex. J Neurosci 26: 12186-12197.

Cowell RA, Bussey TJ, Saksida LM. 2010. Components of recognition memory: dissociable cognitive processes or just differences in representational complexity? Hippocampus 20: 1245-1262.

Crawford JR, Howell DC. 1998. Comparing an individual's test score against norms derived from small samples. Clin Neuropsychol 12: 482-486.

Crawford JR, Garthwaite PH, Azzalini A, Howell DC, Laws KR. 2006. Testing for a deficit in single-case studies: effects of departures from normality. Neuropsychologia 44: 666-677.

Crawford JR, Garthwaite PH, Porter S. 2010. Point and interval estimates of effect sizes for the case-controls design in neuropsychology: rationale, methods, implementations, and proposed reporting standards. Cogn Neuropsychol 27: 245-260.

D'Angelo MC, Kacollja A, Rabin JS, Rosenbaum RS, Ryan JD. 2015. Unitization supports lasting performance and generalization on a relational memory task: evidence from a previously undocumented developmental amnesic case. Neuropsychologia 77: 185-200.

D'Angelo MC, Rosenbaum RS, Ryan JD. 2016. Impaired inference in a case of developmental amnesia. Hippocampus 26: 1291-1302.

Davachi L. 2006. Item, context and relational episodic encoding in humans. Curr Opin Neurobiol 16: 693-700.

Della Sala S, Cowan N, Beschin N, Perini M. 2005. Just lying there, remembering: improving recall of prose in amnesic patients with mild cognitive impairment by minimising interference. Memory 13: 435-440.

Dewar M, Cowan N, Della Sala S. 2007. Forgetting due to retroactive interference: a fusion of Muller and Pilzecker's (1900) early insights into everyday forgetting and recent research on anterograde amnesia. Cortex 43: $616-634$.

Dewar M, Garcia YF, Cowan N, Della Sala S. 2009. Delaying interference enhances memory consolidation in amnesic patients. Neuropsychology 23: $627-634$.

Dewar M, Alber J, Butler C, Cowan N, Della Sala S. 2012. Brief wakeful resting boosts new memories over the long term. Psychol Sci 23: 955-960.

Diana RA, Yonelinas AP, Ranganath C. 2007. Imaging recollection and familiarity in the medial temporal lobe: a three-component model. Trends Cogn Sci 11: 379-386.

Efron B. 1992. Jackknife-after-bootstrap standard errors and influence factions. I R Stat Soc, Ser B 54: 83-127.

Erez J, Cusack R, Kendall W, Barense MD. 2016. Conjunctive coding of complex object features. Cerebral Cortex 26: 2271-2282.

Garrido L, Duchaine B, Nakayama K. 2008. Face detection in normal and prosopagnosic individuals. J Neuropsychol 2: 119-140.

Graham KS, Barense MD, Lee AC. 2010. Going beyond the LTM in the MTL: a synthesis of neuropsychological and neuroimaging findings on the role of the medial temporal lobe in memory and perception. Neuropsychologia 48: 831-853.

Holdstock JS, Mayes AR, Roberts N, Cezayirli E, Issac CL, O'Reilly RC, Norman KA. 2002. Under what conditions is recognition spared relative to recall after selective hippocampal damage in humans? Hippocampus 12: $341-351$.

Kishiyama MM, Yonelinas AP, Kroll NE, Lazzara MM, Nolan EC, Jones EG, Jagust W. 2005. Bilateral thalamic lesions affect recollection- and familiarity-based recognition memory judgments. Cortex 41: 778-788.

Lee AC, Yeung LK, Barense MD. 2012. The hippocampus and visual perception. Front Hum Neurosci 6: 91.

MacMillan N, Creelman C. 2005. Detection theory: a user's guide. Lawrence Erlbaum Associates, Mahwah, NJ.

Martin CB, Bowles B, Mirsattari SM, Köhler S. 2011. Selective familiarity deficits after left anterior temporal-lobe removal with hippocampal sparing are material specific. Neuropsychologia 49: 1870-1878.

Martin CB, McLean DA, Kohler S. 2013. Distinct familiarity-based response patterns for faces and buildings in perirhinal and parahippocampal cortex. J Neurosci 33: 10915-10923.

Mayes AR, Montaldi D, Migo E. 2007. Associative memory and the medial temporal lobes. Trends Cogn Sci 11: 126-135.

McTighe SM, Cowell RA, Winters BD, Bussey TJ, Saksida LM. 2010. Paradoxical false memory for objects after brain damage. Science 330: 1408-1410.
Migo E, Montaldi D, Norman KA, Quamme J, Mayes AR. 2009. The contribution of familiarity to recognition memory is a function of test format when using similar foils. Q J Exp Psychol 62: 1198-1215.

Migo E, Quamme J, Holmes S, Bendell A, Norman KA, Mayes AR, Montaldi D. 2014. Individual differences in forced-choice recognition memory: partitioning contributions of recollection and familiarity. Q J Exp Psychol 67: 2189-2206.

Mitchell AS, Chakraborty S. 2013. What does the mediodorsal thalamus do? Front Syst Neurosci 7: 37.

Monti JM, Balota DA, Warren DE, Cohen NJ. 2014. Very mild Alzheimer's disease is characterized by increased sensitivity to mnemonic interference. Neuropsychologia 59: 47-56.

Murray EA, Wise SP. 2012. Why is there a special issue on perirhinal cortex in a journal called Hippocampus?: the perirhinal cortex in historical perspective. Hippocampus 22: 1941-1951.

Nasseri M, Olsen RK, Martin CB, Rabin JS, Gao FQ, Honjo K, Palombo DJ, Newsome RN, Black SE, Ryan JD, et al. 2017. Impaired face discrimination following early mediodorsal thalamic damage. Paper presented at the International Neuropsychological Society, New Orleans, LA.

Newsome RN, Duarte A, Barense MD. 2012. Reducing perceptual interference improves visual discrimination in mild cognitive impairment: implications for a model of perirhinal cortex function. Hippocampus 22: 1990-1999.

Norman KA. 2010. How hippocampus and cortex contribute to recognition memory: revisiting the complementary learning systems model. Hippocampus 20: $1217-1227$.

Norman KA, O'Reilly RC. 2003. Modeling hippocampal and neocortical contributions to recognition memory: a complementary-learning systems approach. Psychol Rev 110: 611-646.

O'Neil EB, Barkley V, Kohler S. 2013. Representational demands modulate involvement of perirhinal cortex in face processing. Hippocampus 23: 592-605.

Parker RI. 2006. Increased reliability for single-case research results: is the bootstrap the answer? Behav Ther 37: 326-338.

Parker A, Gaffan D. 1998. Interaction of frontal and perirhinal cortices in object recognition memory in monkeys. Eur J Neurosci 10: 3044-3057.

Parker A, Eacott M, Gaffan D. 1997. The recognition memory deficit caused by mediodorsal thalamic lesion in non-human primates: a comparison with rhinal cortex lesion. Eur J Neurosci 9: 2423-2431.

Preacher KJ, Hayes AF. 2008. Asymptotic and resampling strategies for assessing and comparing indirect effects in multiple mediator models. Behav Res Methods 40: 879-891.

Ranganath C. 2010. A unified framework for the functional organization of the medial temporal lobes and the phenomenonlogy of episodic memory. Hippocampus 20: 1263-1290.

Romberg C, McTighe SM, Heath CJ, Whitcomb DJ, Cho K, Bussey TJ, Saksida LM. 2012. False recognition in a mouse model of Alzheimer's disease: rescue with sensory restriction and memantine. Brain 135: 2103-2114.

Ross D, Sadil P, Wilson D, Cowell RA. 2017. Hippocampal engagement during recall depends on memory content. Cereb Cortex. doi: 10.1093/ cercor/bhx147

Rudebeck SR, Scholz J, Millington R, Rohenkohl G, Johansen-Berg H, Lee AC. 2009. Fornix microstructure correlates with recollection but not familiarity memory. J Neurosci 29: 14987-14992.

Ryan JD, D'Angelo MC, Kamino D, Ostreicher M, Moses SN, Rosenbaum RS. 2015. Relational learning and transitive expression in aging and amnesia. Hippocampus 26: 170-184.

Sadeh T, Ozubko J, Winocur G, Moscovitch M. 2014. How we forget may depend on how we remember. Trends Cogn Sci 18: 26-36.

Saunders RC, Mishkin M, Aggleton JP. 2005. Projections from the entorhinal cortex, perirhinal cortex, presubiculum, and parasubiculum to the medial thalamus in macaque monkeys: identifying different pathways using disconnection techniques. Exp Brain Res 167: 1-16.

Smith ML, Gosselin F, Schyns PG. 2012. Measuring internal representations from behavioral and brain data. Curr Biol 22: 191-196.

Squire LR, Wixted JT, Clark R. 2007. Recognition memory and the medial temporal lobe: a new perspective. Nat Rev Neurosci 8: 872-883.

Sun SZ, Fidalgo CO, Barense MD, Lee ACH, Cant JS, Ferber S. 2017. Erasing and blurring memories: the differential impact of interference on separate aspects of forgetting. J Exp Psychol Gen 146: 1606-1630.

Tingley D, Yamamoto T, Hirose K, Keele L, Imai K. 2014. Mediation: R package for causal mediation analysis. J Stat Softw 59: 1-38.

Tombaugh T. 2004. Trail making test A and B: normative data stratified by age and education. Arch Clin Neuropsychol 19: 203-214.

Tombaugh T, Kozak J, Rees L. 1999. Normative data stratified by age and education for two measures of verbal fluency: FAS and animal naming. Arch Clin Neuropsychol 14: 167-177.

Trelle AN, Henson RN, Green DAE, Simons JS. 2017. Declines in representational quality and strategic retrieval processes contribute to 
Impaired forced choice with intact yes/no recognition memory

age-related increased in false recognition. J Exp Psychol Learn Mem Cogn. doi: 10.1037/xlm0000412.

Tsivilis D, Vann SD, Denby C, Roberts N, Mayes AR, Montaldi D, Aggleton JP. 2008. A disproportionate role for the fornix and mammillary bodies in recall versus recognition memory. Nat Neurosci 11: $834-842$.

Vann SD, Denby C, Love S, Montaldi D, Renowden S, Coakham HB. 2008. Memory loss resulting from fornix and septal damage: impaired supra-span recall but preserved recognition over a 24-hour delay. Neuropsychology 22: 658-668.

Vann SD, Tsivilis D, Denby C, Quamme J, Yonelinas AP, Aggleton JP, Montaldi D, Mayes AR. 2009. Impaired recollection but spared familiarity in patients with extended hippocampal system damage revealed by 3 convergent methods. Proc Natl Acad Sci 106: 5442-5447.

Vargha Khadem F, Gadian D, Watkins K, Connelly A, Van Paesschen W, Mishkin M. 1997. Differential effects of early hippocampal pathology on episodic and semantic memory. Science 277: 376-380.

Vargha-Khadem F, Salmond CH, Watkins K, Friston KJ, Gadian D, Mishkin M. 2003. Developmental amnesia: effect of age at injury. Proc Natl Acad Sci 100: 10055-10060.
Watson HC, Lee AC. 2013. The perirhinal cortex and recognition memory interference. J Neurosci 33: 4192-4200.

Westerberg CE, Mayes AR, Florczak SM, Chen Y, Creery J, Parrish T, Weintraub S, Mesulam MM, Reber PJ, Paller KA. 2013. Distinct medial temporal contributions to different forms of recognition in amnestic mild cognitive impairment and Alzheimer's disease. Neuropsychologia 51: $2450-2461$.

Wixted JT. 2004. The psychology and neuroscience of forgetting. Annu Rev Psychol 55: 235-269.

Yeung LK, Ryan JD, Cowell RA, Barense MD. 2013. Recognition memory impairments caused by false recognition of novel objects. J Exp Psychol Gen 142: 1384-1397.

Yonelinas AP. 2002. The nature of recollection and familiarity: a review of 30 years of research. J Mem Lang 46: 441-517.

Zola-Morgan S, Squire LR. 1985. Medial temporal lesions in monkeys impair memory in a variety of tasks sensitive to human amnesia. Behav Neurosci 99: $22-34$.

Received March 7, 2018; accepted in revised form October 2, 2018. 


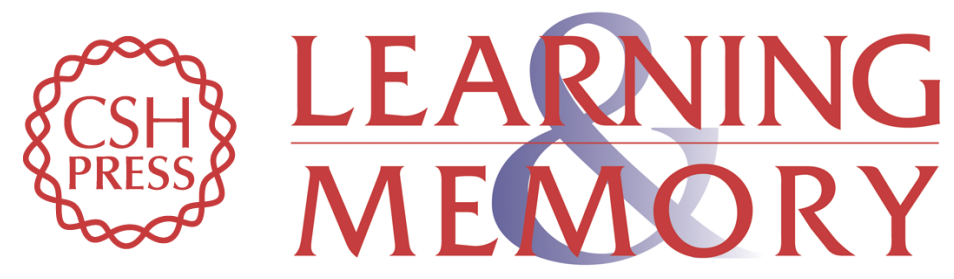

\section{Dissociable contributions of thalamic nuclei to recognition memory: novel evidence from a case of medial dorsal thalamic damage}

Rachel N. Newsome, Alexandra N. Trelle, Celia Fidalgo, et al.

Learn. Mem. 2018, 25:

Access the most recent version at doi:10.1101//m.045484.117

References This article cites 79 articles, 9 of which can be accessed free at: http://learnmem.cshlp.org/content/25/1/31.full.html\#ref-list-1

Creative This article is distributed exclusively by Cold Spring Harbor Laboratory Press for the Commons first 12 months after the full-issue publication date (see

License http://learnmem.cshlp.org/site/misc/terms.xhtml). After 12 months, it is available under a Creative Commons License (Attribution-NonCommercial 4.0 International), as described at http://creativecommons.org/licenses/by-nc/4.0/.

Email Alerting Receive free email alerts when new articles cite this article - sign up in the box at the Service top right corner of the article or click here. 